\title{
METODOLOGÍA DE CONTROL DE MÁQUINAS ELÉCTRICAS DE IP CON CAPACIDAD DE DEBILITAMIENTO DE CAMPO
}

\section{CONTROL METHODOLOGY OF PM MACHINE WITH FIELD WEAKENING CAPABILITY}

\author{
Delvis González L. ${ }^{1} \quad$ Juan Tapia L. ${ }^{1} \quad$ Roger Wallace C. ${ }^{1}$ \\ Recibido 27 de junio de 2006, aceptado 12 de diciembre de 2006 \\ Received: June 27, 2006 Accepted: December 12, 2006
}

\begin{abstract}
RESUMEN
En el mundo actual los requerimientos de los accionamientos se hacen cada vez más exigentes en cuanto a precisión y eficiencia. Por esta razón los diseñadores se ven obligados a encontrar nuevas topologías y mecanismos de control que las satisfagan, especialmente para aplicaciones de velocidad variable como tracción eléctrica. Las máquinas sincrónicas de imanes permanentes son una solución atractiva debido a su elevada eficiencia y su gran densidad de potencia. En estas aplicaciones, para velocidad por debajo de la nominal, el accionamiento opera a torque constante siguiendo la trayectoria de máximo par por ampere. Para valores de velocidad por encima de la nominal es necesario inyectar un flujo negativo en el eje directo, para reducir el flujo impuesto por el imán. De esta manera el voltaje y la corriente se mantienen en 1.0 pu. Este efecto se logra con un control adecuado del ángulo de la corriente en los ejes $d q$. Este trabajo muestra un método de control para operar las máquinas de corriente alterna e imanes permanentes con capacidad de debilitamiento de campo. Utilizando el Simulink del Matlab se verifica la estrategia de control propuesta. Además se muestran las propiedades de una nueva topología de MCAIP para aplicaciones de velocidad variable.
\end{abstract}

Palabras clave: Máquinas de imanes permanentes, máquinas de flujo axial, capacidad de debilitamiento de campo.

\section{ABSTRACT}

Presently the AC machine drive's requirements are more demanding in terms of precision and efficiency. Under these circumstances, designers are forced to find new machine topologies and control methods to meet these conditions, especially for variable speed applications such us electric traction. PM machines are an attractive solution because of their intrinsic higher efficiency and their power density. For these operations - below the rated speed - the drive works under constant torque strategy following the maximum torque per ampere trajectory. For speeds over the rated value, it is necessary to inject a negative d-axis flux, to reduce the field imposed by the PM. In this manner, voltage and current are maintained in 1.0 per unit values, this effect obtained by appropriated control of the stator current angle in the dq-axis. This paper shows a control method to operate an AC machine with permanent magnet (MACPM) and flux weakening capability. Simulink by Matlab, is used to verify the control strategy. In addition, a new topology of a MACPM for variable speed application is presented and their properties are discussed.

Keywords: Permanent magnet machines, axial flux, field weakening capability.

\section{INTRODUCCIÓN}

Las máquinas eléctricas de corriente alterna e imanes permanentes (MCAIP) han llamado la atención de los diseñadores en los últimos años debido a su elevada eficiencia y densidad de potencia. Debido a que estas son máquinas sincrónicas, necesitan para su funcionamiento algún elemento externo para su partida.
El desarrollo alcanzado en la electrónica de potencia y en las técnicas de control promueve la utilización de las MCAIP con su respectivo convertidor de frecuencia, logrando además un excelente comportamiento de accionamiento en estado estable.

En muchas aplicaciones de alto torque y baja velocidad las MCAIP son las preferidas, pero presentan una particular

\footnotetext{
1 Departamento de Ingeniería Eléctrica, Universidad de Concepción. Casilla 160-C, Correo 3. Concepción, Chile. Email: degonzalez@udec.cl
} 
desventaja para rangos de velocidad por encima de la nominal [1]. El flujo fijo impuesto por los imanes en el entrehierro hace que al aumentar la velocidad, el voltaje inducido en los devanados se incremente a valores muy por encima de los permitidos por la propia máquina y el convertidor.

Para evitar este fenómeno se debe debilitar el campo en el entrehierro para rangos de operación por encima de la velocidad nominal [4]. Numerosos métodos de debilitamiento de campo han aparecido para solucionar este problema. En este trabajo se detalla uno en particular. Utilizando la Técnica de Control Vectorial se coloca el vector de corriente de estator de forma tal que, para velocidades por encima de la nominal, una parte del flujo asociado a ella se reste al flujo principal impuesto por el imán manteniendo el voltaje constante en los terminales de la máquina. Basado en esta técnica, la selección de los parámetros resulta fundamental para extender la capacidad de potencia por sobre la velocidad nominal.

\section{RESTRICCIONES DEL CONVERTIDOR}

El análisis del control parte por el modelo en por unidad de la MCAIP en ejes $d q$ como el mostrado en la figura 1 .

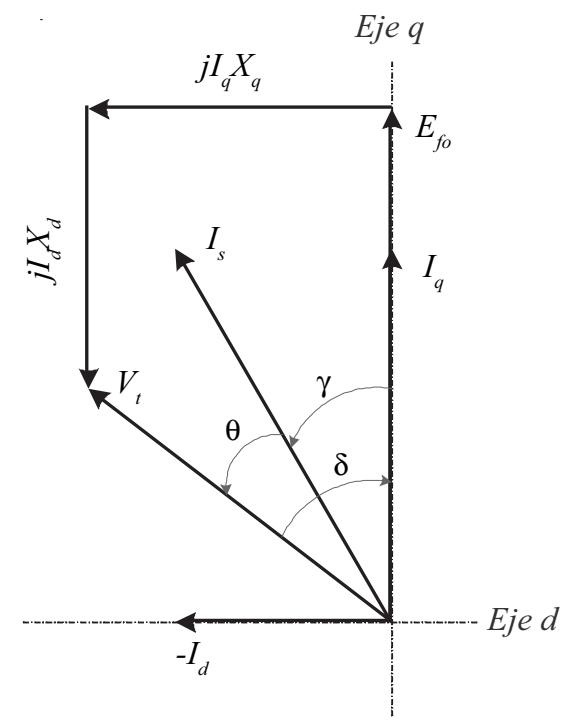

Figura 1. Efecto desmagnetizante y modelo $d q$ de la MCAIP.

Los valores máximos permisibles de corriente y voltaje determinados por el convertidor son:

$$
\begin{gathered}
I_{s}=\sqrt{I_{d}^{2}+I_{q}^{2}} \leq 1 p u \\
V_{s}=\sqrt{V_{d}^{2}+V_{q}^{2}} \leq 1 p u
\end{gathered}
$$

En un sistema de ejes $I_{d}$ e $I_{q}$ en $p u$, el lugar geométrico de los límites de voltaje y corriente son una elipse y una circunferencia respectivamente, tal como se muestra en la figura 2. Para el caso $E_{f 0}=X_{d}=0.7 p u$ y $X_{q 0}=1 p u$. La operación del motor está limitada entonces a la zona donde se cumplen ambas restricciones, la corriente igual a 1.0 pu y la elipse de voltaje, según [3] y [4].

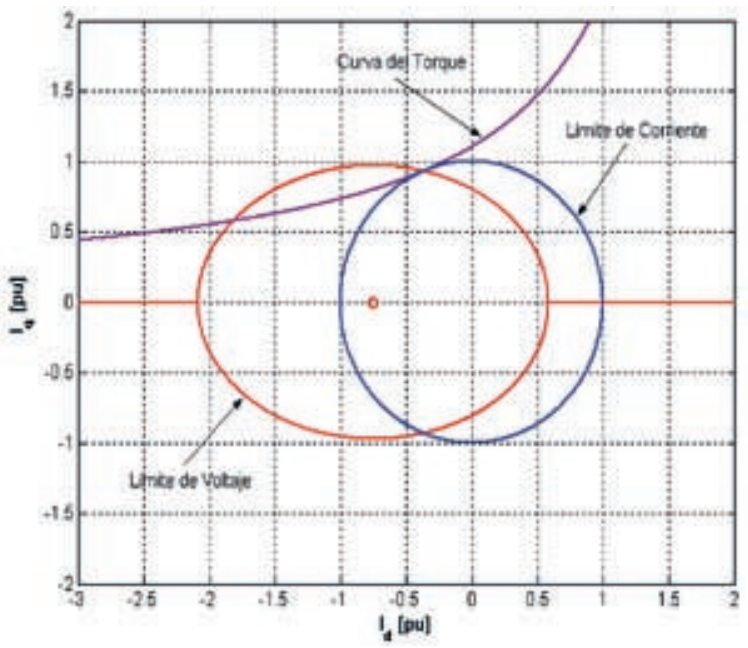

Figura 2. Representación en ejes $d q$ de los valores límites de voltaje y corriente del inversor.

Estas restricciones se pueden cumplir por un número infinito de puntos, de los cuales depende el torque entregado por la máquina, por lo que es necesario conocer cual será la trayectoria de máximo torque por ampere que se puede alcanzar dentro del área disponible.

La ecuación del torque en función del ángulo de corriente $(\gamma)$ es:

$$
T=E_{f o} I_{s} \cos \gamma+\left(\frac{X_{q 0}-X_{d 0}}{2}\right) I_{d}^{2} \sin 2 \gamma
$$

Derivando respecto a $\gamma$ e igualando a cero se llega a:

$$
\sin \gamma^{*}=\frac{-E_{f 0}+\sqrt{E_{f 0}^{2}-8\left(X_{q 0}-X_{d 0}\right)^{2} I_{s}^{2}}}{4\left(X_{q 0}-X_{d 0}\right) I_{s}}
$$


En la ecuación (4) se define el ángulo de corriente óptimo que divide en sus respectivas componentes de corriente en los ejes $d q$ que maximizan el torque por ampere en la máquina.

\section{METODOLOGÍA DE CONTROL}

Siguiendo las restricciones de voltaje y corriente impuestas por el convertidor, la estrategia de control a seguir se puede dividir en dos etapas. En la primera, por debajo de la velocidad nominal, se operará la máquina a torque constante siguiendo la trayectoria de máximo torque por ampere.

En esta etapa la $I_{s}=1.0 \mathrm{pu}$, mientras que el voltaje depende de la velocidad llegando a 1.0 pu para velocidad nominal.

Los valores óptimos de $I_{d}$ e $I_{q}$ en función de la velocidad se obtienen siguiendo la ecuación (4), para $I_{s}=1.0 \mathrm{pu}$ como:

$$
\begin{gathered}
I_{d 1}=\frac{E_{f 0}}{4\left(X_{q 0}-X_{d 0}\right)}-\sqrt{\left(\frac{E_{f 0}}{X_{q 0}-X_{d 0}}\right)^{2}+\frac{1}{2}} \\
I_{q 1}=\sqrt{I_{s}^{2}-I_{d 1}^{2}}
\end{gathered}
$$

La segunda etapa, por encima de la velocidad nominal, el vector de corriente se controlará siguiendo el control de debilitamiento de campo. En ella se pondrá la componente de corriente necesaria en el eje directo, en sentido contrario al flujo del imán, para mantener el voltaje en los terminales de la máquina en $1.0 \mathrm{pu}$.

Así, controlando el flujo de reacción de armadura, se puede operar la máquina a velocidades por encima de la velocidad nominal. Las componentes de corriente en los ejes $d q$ que producen potencia máxima están dados por:

$$
\begin{gathered}
I_{d 2}=\frac{X_{d 0} E_{f 0}}{X_{q 0}^{2}-X_{d 0}^{2}} \\
-\frac{\sqrt{\left(X_{d 0} E_{f 0}\right)^{2}+\left(X_{q 0}^{2}-X_{d 0}^{2}\right)\left(E_{f 0}^{2}+X_{q 0}^{2}-1 / w^{2}\right)}}{X_{q 0}^{2}-X_{d 0}^{2}} \\
I_{q 2}=\sqrt{I_{s}^{2}-I_{d 2}^{2}}
\end{gathered}
$$

En estas condiciones $I_{d 2}$ se acerca a -1.0 mientras que $I_{q 2}$ tiende a cero, punto en el cual la potencia también se hace cero. Esto ocurre a una velocidad $w_{c}$, dada por:

$$
w_{c}=\frac{1}{E_{f 0}-X_{d 0}}
$$

De la expresión (9) se deduce que mientras más cerca estén los valores en $p u$ de $E_{f 0}$ y $X_{d 0}$ mayor será el rango de operación de la máquina por encima de la velocidad nominal, y para la condición de $E_{f 0}=X_{d 0}$ se puede aumentar infinitamente la velocidad sin que la potencia se anule. Para esta condición, el comportamiento de la potencia y el torque se muestran en la figura 3, mientras que en la figura 4 se muestra la evolución de las corrientes en los ejes $d q$.

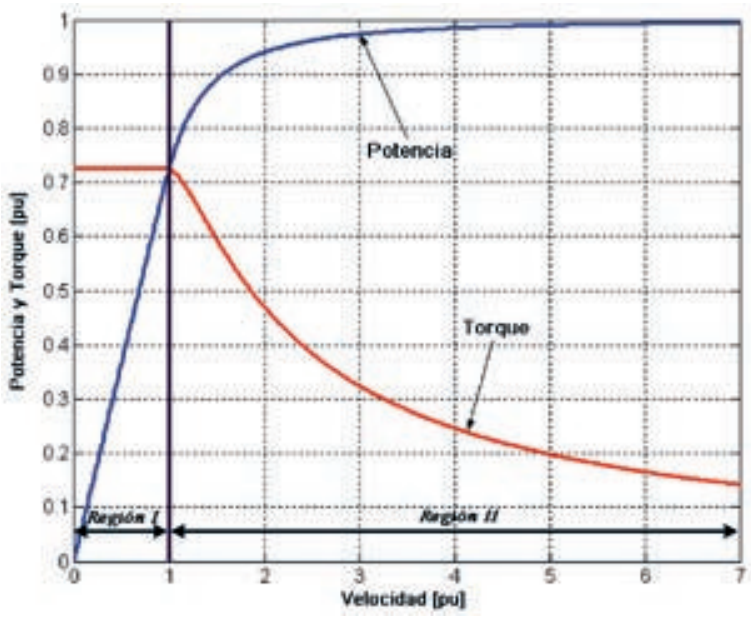

Figura 3. Comportamiento de la Potencia y el Torque para $E_{f 0}=X_{d 0}=0.7, X_{q 0}=1.0$.

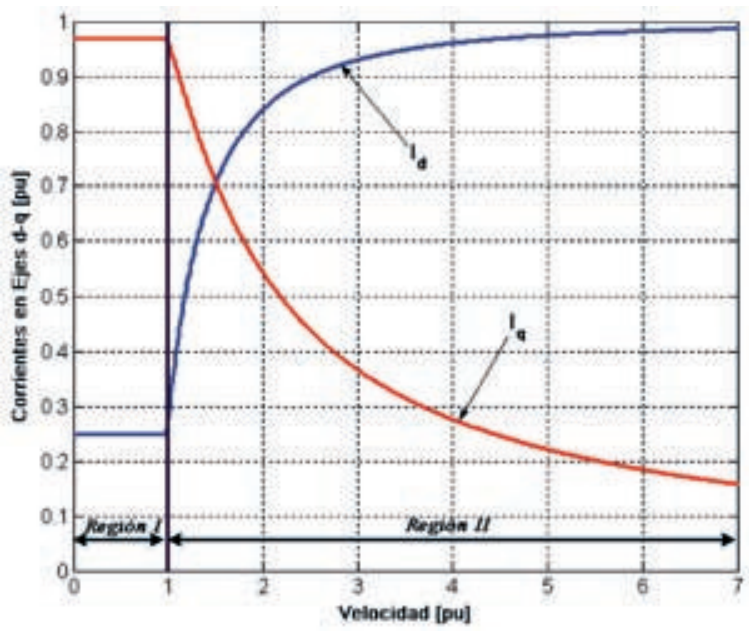

Figura 4. Comportamiento de las corrientes de ejes $d q$ para $E_{f 0}=X_{d 0}=0.7, X_{q 0}=1.0$. 


\section{ESQUEMA DE CONTROL}

El esquema de control a desarrollar, tanto para la etapa de torque constante como para la de debilitamiento de campo se basa en el análisis realizado. Este se muestra en la figura 5, donde el torque de referencia $T^{*}$ se genera a partir del error de velocidad. Dependiendo del modo de operación $T^{*}$ es procesado por el bloque de la etapa 1 o la 2. La primera corresponde a la región de operación de torque constante y la segunda a la de debilitamiento de campo [2].

A la salida de estos bloques se tendrán las referencias de $I_{d}{ }^{*}$ e $I_{q} *$ a seguir para generar el máximo torque por ampere para cualquier velocidad de operación menor o igual a la nominal y, para valores mayores de velocidad, proporcionan en flujo negativo en eje directo necesario para mantener el voltaje terminal en $1.0 \mathrm{pu}$ con la potencia máxima de salida.

Con $I_{d}{ }^{*} \mathrm{e} I_{q}^{*}$ y el ángulo de posición del rotor se generan entonces las corrientes de fase necesarias para lograr el comportamiento óptimo del accionamiento.

\section{SIMULACIONES}

Siguiendo esta metodología de control se simula el control de un motor sincrónico con imanes permanentes. Utilizando los modelos de las máquinas que ofrece el Power System Blockset, en el Simulink del Matlab, se crea el esquema de control explicado y, con una selección adecuada de los parámetros de la máquina, se logran comportamientos del conjunto accionamiento-motor similares a los teóricos para el rango de velocidad desde 0 hasta $2.7 p u$.

Como velocidad de referencia se aplicó una rampa de tal forma que, después de la partida, siguiera aumentando hasta valores superiores a la velocidad nominal. Esta se compara entonces con una muestra de la velocidad del rotor y el resultado se introduce a un controlador proporcional-integral que dará la $I_{q}{ }^{*}$ para la primera etapa del control.

Los valores de $I_{d}{ }^{*}$ para todo el rango de operación y los de $I_{q}^{*}$ para la segunda etapa se obtienen a partir de una Look up Table en función de la velocidad del rotor. Estas tablas están parametrizadas con los vectores de $I_{d}$ e $I_{q}$ cuyo comportamiento depende de los parámetros de diseño de la máquina. De la misma forma se puede obtener el comportamiento de cualquier diseño dado por la relación de parámetros $X_{d}, X_{q}$ y $E_{f 0}$, deseada.

Con las referencias de $I_{d}{ }^{*}, I_{q}{ }^{*}$ y $\theta_{r}$ se generan entonces los patrones de corrientes de fase a entrar al inversor PWM para generar los voltajes de línea a aplicar al motor.

Para una relación adecuada de $X_{d}, X_{q}$ y $E_{f 0}$ se obtuvieron las gráficas que se muestran a continuación, donde la $w_{c}=2.7 \mathrm{pu}$.

En la figura 6 se muestra el comportamiento de la velocidad del rotor y del torque en función del tiempo. La velocidad llega hasta $2.7 p u$ mientras que el torque permanece constante en la primera etapa; una vez que se alcanza la velocidad nominal comienza a disminuir hasta que se hace prácticamente cero a la velocidad $w_{c}$.

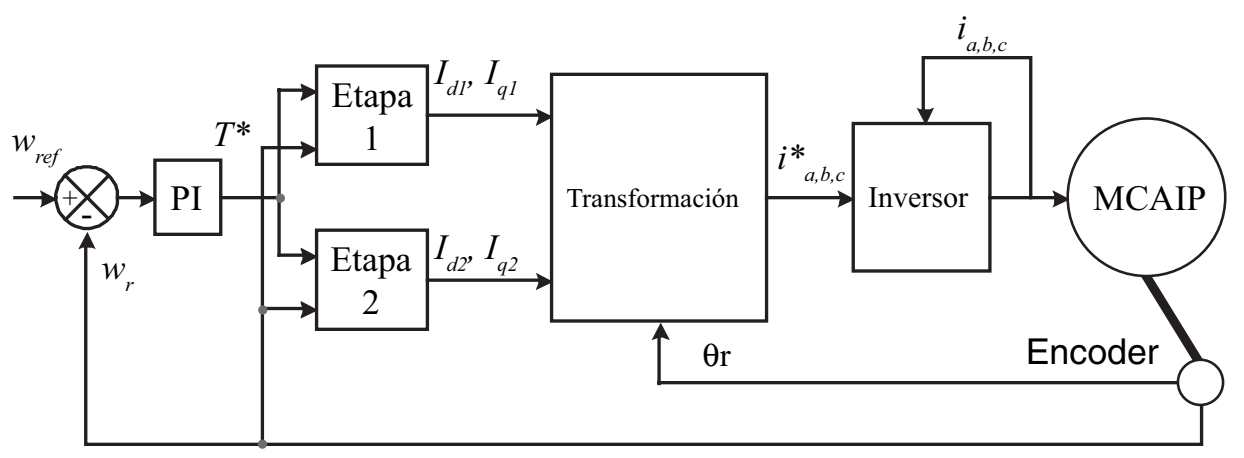

Figura 5. Esquema de Control. 

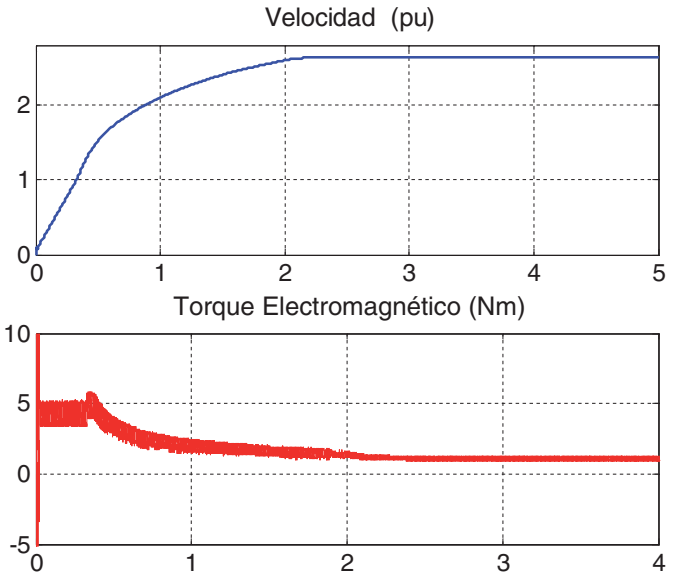

Figura 6. Comportamiento de la velocidad y el torque en función del tiempo.

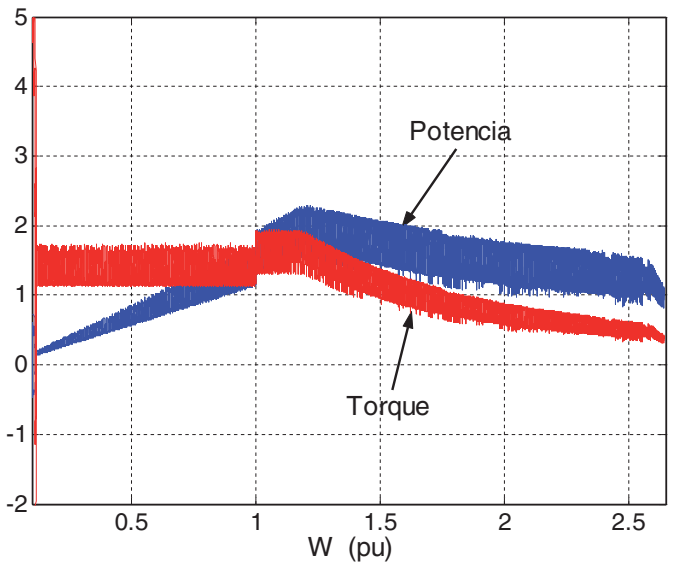

Figura 7. Comportamiento del torque y la potencia en función de $w_{p u}$.

Esta misma evolución presenta el torque en la figura 7 , ahora en función de la velocidad en $p u$. Se muestra también la potencia entregada por la máquina que toma un valor máximo y después tiende a cero a medida que se alcanza la velocidad $w_{c}$. En ambos casos se observan los transientes propios de la partida de la máquina.

Los gráficos de la figura 8 describen la evolución de las corrientes en los ejes $d q$, primero en función del tiempo y después en función de la velocidad del motor en por unidad. En ambos casos la $I_{d}$ es casi cero para la primera etapa, mientras que casi toda la corriente está sobre el eje de cuadratura para lograr la trayectoria de máximo torque por ampere. Recordemos que la componente de corriente en el eje $q$ es quien provoca torque en la máquina.
Una vez que se alcanza la velocidad nominal es necesario reducir el flujo en el entrehierro, por lo que comienza a aumentar la corriente en sentido contrario en el eje directo a medida que la velocidad crece. Cuando se llega a la velocidad $w_{c}$ prácticamente toda la corriente está en el eje directo, mientras que en el eje de cuadratura es casi cero. Por esta razón el torque en este punto se hace cero.
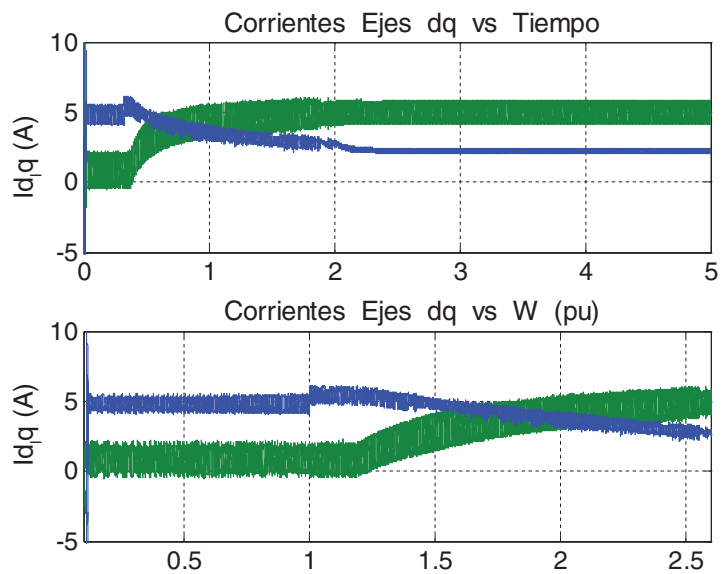

Figura 8. Evolución de las corrientes en ejes $d q$ en función del tiempo y de $w_{p u}$.

\section{MÁQUINAS CON CAPACIDAD DE DEBILITAMIENTO DE CAMPO}

Los imanes permanentes tienen una permeabilidad semejante a la del aire, por lo que el flujo de reacción de armadura tendría que cruzar una distancia igual a la longitud del entrehierro y además, la altura del imán. Esta trayectoria impone una reluctancia demasiado alta para lograr el debilitamiento deseado, por lo que el gran problema, desde el punto de vista de diseño, está en ofrecer al flujo de reacción de armadura un camino de baja reluctancia para que pueda cruzar el entrehierro [4]. Para lograr este objetivo los diseñadores han propuesto varias configuraciones de máquinas eléctricas.

Nuestro grupo de trabajo también se ha dirigido en esa línea de investigación, proponiendo variantes de diseño de máquinas de flujo axial e imanes permanentes con capacidad de debilitamiento de campo. Una topología propuesta se muestra en la figura 9. Esta es una máquina de flujo axial e IP con un estator central y dos rotores, donde se encuentran montados los imanes. 


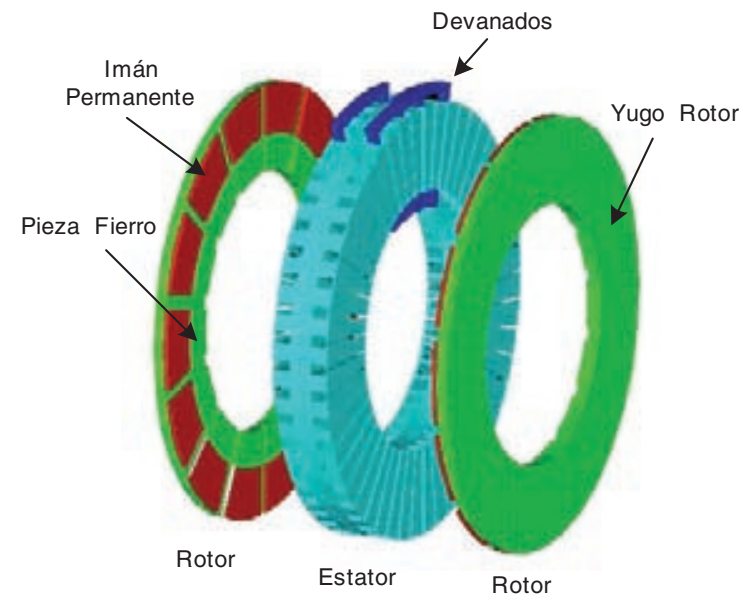

Figura 9. Máquina de flujo axial e IP con capacidad de debilitamiento de campo.

Una manera eficaz de lograr que una gran parte del flujo de reacción de armadura cruce el entrehierro es sustituir una porción del imán del rotor por una pieza de hierro. De esta forma el flujo inyectado por el devanado del estator en sentido contrario se restará en el entrehierro y circulará al rotor a través del hierro. En la figura 10 se observa un polo del rotor. El diseño y la optimización se realizan a partir de simulaciones tridimensionales basadas en el Método de Elementos Finitos (MEF), con ayuda del Software profesional Flux3D.

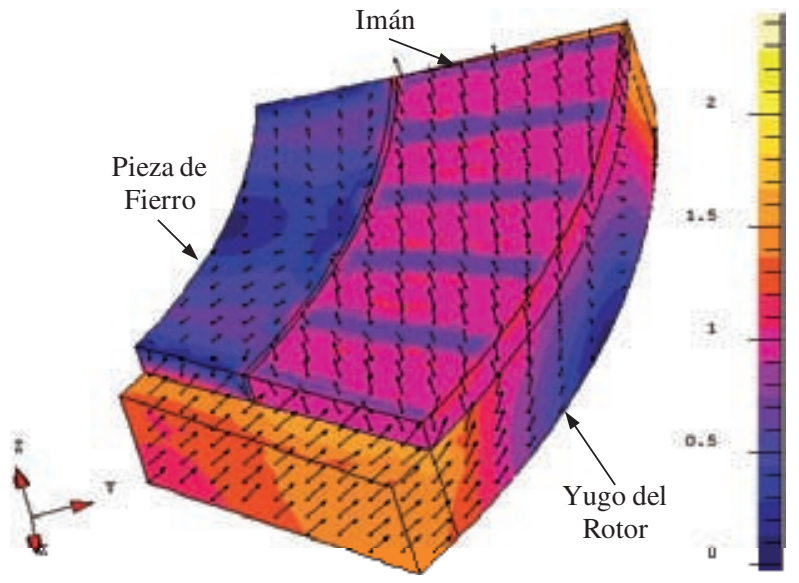

Figura 10. Máquina de flujo axial y capacidad de debilitamiento de campo.

En la topología mostrada, para una razón del área de hierro al área del imán de 0.47 se puede reducir el flujo en función de los ampare-vueltas como se muestra en la figura 11 .
Evidentemente a medida que aumentamos la relación entre el área del hierro y del imán, se incrementa la capacidad de debilitamiento del campo, pero se disminuye el flujo en el entrehierro. Esto trae consigo la disminución del torque y la potencia que es capaz de entregar la máquina.

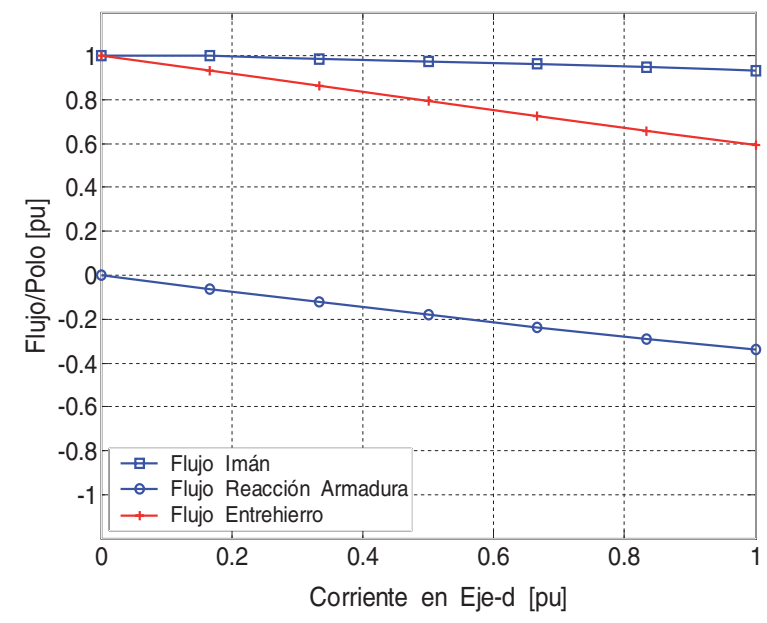

Figura 11. Capacidad de debilitamiento del campo en función de los ampere-vueltas.

Se hace necesario entonces llegar a un compromiso entre el debilitamiento deseado y la potencia de salida de la máquina. En base a ello, la figura 12 muestra el comportamiento del flujo resultante en el entrehierro en función de la corriente en el eje- $d$ (en $p u$ ) para relaciones de Hierro/Imán que van desde 0.2 hasta 0.6. Este será un factor determinante en el diseño de la máquina para lograr el comportamiento deseado.

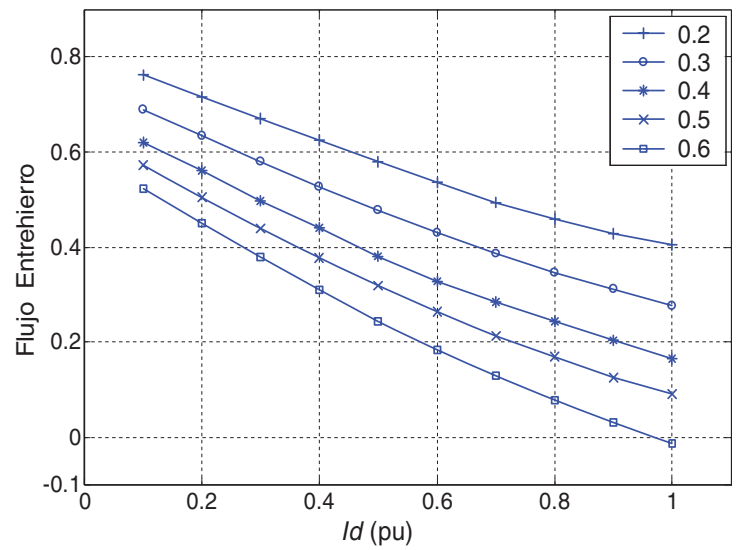

Figura 12. Capacidad de debilitamiento vs Id para Distintas relaciones Fierro/Imán.

Esta variante disminuye el riesgo de desmagnetización del imán por someterlo a un flujo intenso en sentido contrario. 


\section{CONCLUSIONES}

En el procedimiento matemático antes descrito se parte de varios supuestos como despreciar la resistencia de armadura y no considerar el coeficiente de fricción de la máquina entre otros, que sí son considerados en el modelo de la máquina propuesto en el Matlab, por lo que los resultados no son exactamente iguales a los teóricos, pero sí describen un comportamiento similar y esperado del conjunto inversor-motor.

En el accionamiento simulado se logra operar una MCAIP por encima de su velocidad nominal. En el ejemplo mostrado se puede sobrepasar la velocidad por encima de 2.5 veces su valor nominal cumpliendo las restricciones impuestas por el convertidor, lo que prueba la validez del esquema de control.

A partir del modelo de la máquina usando el MEF se obtienen los valores de los parámetros de diseño $\left(X_{d}\right.$, $X_{q}$ y $\left.E_{f 0}\right)$, necesarios para correr las simulaciones en MATLAB.

Esta metodología de control demuestra que la capacidad de potencia de la máquina por encima de la velocidad nominal depende básicamente de los parámetros de diseño. Calculando adecuadamente los valores de $X_{d}$, $X_{q}$ y $E_{f 0}$ se puede extender el rango de operación de una MCAIP a valores deseados. Se debe tener en cuenta que el modelo empleado en la simulación no considera la saturación del circuito magnético, por lo que los valores de reactancias permanecen invariables. En la práctica este es un punto a considerar, pues a medida que aumenta el flujo varía el valor de $X_{d}$ y los resultados pueden variar.

\section{NOMENCLATURA}

$I_{d}, I_{q} \quad:$ Corrientes de eje directo y cuadratura.

$V_{d}, V_{q} \quad$ : Voltajes de eje directo y cuadratura.

$n_{p u} \quad:$ Velocidad en pu.

$E_{f 0} \quad:$ Tensión a circuito abierto y a velocidad nominal.

$X_{d 0}, X_{q 0}:$ Reactancias de eje directo y cuadratura a

velocidad nominal

$\delta \quad:$ Ángulo de torque.

$\gamma \quad$ : Ángulo de corriente.

$T^{*} \quad:$ Torque de referencia.

$I_{d}{ }^{*}, I_{q}^{*} \quad$ : Corrientes de eje dq de referencia.

$w_{\text {ref }}$ : Velocidad de referencia.

$\theta_{r}$ : : Ángulo de posición del rotor.

\section{REFERENCIAS}

[1] T. Jahns. "Motion Control with Permanent-Magnet AC Machine". Proceeding of the IEEE. Vol. 82 No 8. August 1986.

[2] R. Krishnan, R. "Electric Motor Drives". Segunda Edición, Prentice Hall. 2001.

[3] N. Bianchi, S. Bolognani y B. j. Chalmers. "Salient Rotor PM Synchronous Motors for an Extended Flux-Weakening Operation Range". IEEE Transactions on Industry Applications. Vol. $36 \mathrm{~N}^{\circ}$ 4. Jul/Aug 2000.

[4] J. Tapia. "Development of the Consequent Pole Permanent Magnet Machine". Preliminary Exam submitted in partial fulfilment of the requirements for the degree of Doctor of Philosophy. University of Wisconsin - Madison. 2001. 